\title{
Localization and spectral statistics in a banded random matrix ensemble
}

\author{
Michael Wilkinson $\dagger$, Mario Feingold $\ddagger \|$ and David M Leitner $\$$ \\ $\ddagger$ Department of Physics and Applied Physics, John Anderson Building, University of \\ Strathclyde, Glasgow, G4 0NG, Scotland, UK \\ ¥ Lawrence Berkeley Laboratory and Department of Physics, University of California, \\ Berkeley, CA 94720 , USA \\ $\S$ Department of Chemistry, Brown University, Providence, RI 02912, USA
}

Received 4 July 1990

\begin{abstract}
We investigate the localization properties of the eigenvectors of a banded random matrix ensemble, in which the diagonal matrix elements increase along the diagonal. We relate the results to a transition in the spectral statistics which is observed as a parameter is varied, and discuss the relevance of this model to the quantum mechanics of chaotic Hamiltonian systems.
\end{abstract}

\section{Introduction}

It is by now well established that random matrix ensembles can provide a useful statistical model for the fine detail in the spectrum of quantum systems which have a chaotic classical limit: in particular the Gaussian orthogonal ensemble (GOE) (Porter 1965) provides an excellent model for systems with real matrix elements (Bohigas, Giannoni and Schmidt 1984). Theoretical arguments (Berry 1977) suggest that the eigenfunctions of these systems can be modelled by a Gaussian random function with a known autocorrelation function, and numerical experiments support the view that this picture is essentially correct (Sebr 1990), although there are additional features which correlate with short period classical orbits (Heller 1984). If the eigenfunctions are quasi-random, then so are the matrix elements of any operator which uses these states as a basis: this hypothesis is obviously very important in modelling the dynamical response of chaotic quantum systems. In this paper we test the consistency of the idea that the matrix elements can be modelled by a random matrix: we set up a random matrix model which mimics the matrix elements of a chaotic Hamiltonian $\hat{H}=\hat{H}_{0}+\hat{H}_{1}$, expressed in the basis formed by the eigenstates of another chaotic Hamiltonian $\hat{H}_{0}$. If modelling the matrix by random variables is justified, we expect the spectral statistics of our random matrix model to be of GOE type, in agreement with known results. We find that this is not always the case, and conclude that assumptions about the randomness of the matrix elements, while they are a very useful approximation, should be used with caution. 
Consider the matrix elements of $\hat{H}=\hat{H}_{0}+\hat{H}_{1}$ in the basis formed by the (energy ordered) eigenstates $|i\rangle|j\rangle \ldots$ of $\hat{H}_{0}:\langle i|\hat{H}| j\rangle=E_{i} \delta_{i j}+\left\langle i\left|\hat{H}_{1}\right| j\right\rangle$, where $E_{i}$ are eigenvalues of $\hat{H}_{0}$. These matrix elements are small if $\left|E_{\mathrm{i}}-E_{j}\right| \gg \hbar / \tau$, where $\tau$ is a classical timescale characterizing the autocorrelation of $H_{1}$ under the dynamics of $H_{0}$ (Feingold and Peres 1986, Wilkinson 1987). We can model this matrix using a random matrix ensemble with the following properties: the matrix elements are independently gaussian distributed, so that the ensemble is defined by giving the mean and variance of the distributions of the matrix elements $H_{i j}$ :

$$
\begin{aligned}
& \left\langle H_{i j}\right\rangle=\alpha i \delta_{i j} \\
& \left\langle\left(H_{i j}-\left\langle H_{i j}\right\rangle\right)^{2}\right\rangle= \begin{cases}1+\delta_{i j} & |i-j|<b \\
0 & |i-j| \geqslant b\end{cases}
\end{aligned}
$$

i.e., the matrix is banded, with $2 b-1$ elements in each row, and with the mean of the diagonal matrix elements increasing along the diagonal. The parameters $\alpha$ and $b$ are related to the density of states $\rho$ of $H_{0}$ and typical size $\sigma$ of the matrix elements of $H_{1}$ by $b=\rho \hbar / \tau, \alpha=1 / \rho \sigma$. The dimension $\mathcal{N}$ of the matrix is assumed to be very large, and to eliminate end effects it will become clear that $\mathcal{N}$ should satisfy $\mathcal{N} \gg b^{2}$. We discuss the spectral statistics of this model, which undergo a transition from Poisson to GOE statistics as a parameter is varied. This transition depends on the fact that the eigenvectors of the matrix exhibit Anderson localization, and much of the technical content of this paper is concerned with estimating the localization length. The method used to estimate the localization length is an adaptation of an argument used by Chirikov et al (1981) for a unitary operator: we believe that it may be useful for both analytical and numerical studies of other localization problems involving hermitean operators. We note that a very similar random matrix ensemble was studied by Wigner (1955), whose analysis was confined to a study of the density of states. A more detailed discussion of the spectral statistics of our model and its semiclassical significance will be published elsewhere (Feingold et al 1990).

\section{Spectral statistics of the model}

First we consider the special case in which $\alpha=0$, where the model is already well understood. When $b=\mathcal{N}$, the model becomes the Gaussian orthogonal ensemble (GOE), and exhibits level repulsion: in particular the level spacing distribution $P(S)$ is very similar to the Wigner distribution (Porter 1965). On the other hand when $b \ll \mathcal{N}$ our model is a banded random matrix, and a generalization (Johnson and Kunz 1983) of the Furstenberg theorem (Ishii 1973) implies that the eigenvectors are localized, with some characteristic localization length $L$. When $L \ll \mathcal{N}$, this implies that level repulsion does not affect the level spacing distribution $P(S)$, because eigenfunctions localized in regions separated by more than a localization length would not experience level repulsion. In this case it is clear that $P(S)$ must be a Poisson distribution (Molcanov 1981). When $L \simeq \mathcal{N}$ there is a crossover between Poisson and Wigner statistics, which has been characterized in a finite-size scaling picture by Casati et al (1990).

Now consider what happens when $\alpha$ is non-zero. An eigenvector $\phi_{i}$ which is localized so that its dominant contributions are around the $i$ th element will have an energy $E_{i}$ which is close to the value of the $i$ th diagonal matrix element, with mean 
value $\alpha i$. The difference between $E_{\mathrm{i}}$ and $\alpha i$ can be estimated by regarding the eigenvectors as quasirandom, so that the off-diagonal terms contribute incoherently, hence

$$
\left|E_{i}-\alpha i\right|=\mathrm{O}(\sqrt{b}) \text {. }
$$

Note that this estimate is consistent with the Wigner semicircle law (Porter, 1965) for the density of states of the GOE. States which are separated by a distance of the order of the localization length are expected to exhibit level repulsion if they have nearly equal energies, but states separated by many localization lengths cannot experience level repulsion. If $\alpha$ is sufficiently large, (2.1) shows that two states separated by more than a localization length are very unlikely to have similar energies, so that all pairs of states of similar energy exhibit level repulsion, and the level spacing distribution is therefore of the Wigner type. The condition for this is:

$$
\gamma=\frac{\alpha L}{\sqrt{b}} \gg 1
$$

When $\gamma \ll 1$ on the other hand, states separated by several localization lengths can have similar energies, and we expect a transition to Poisson statistics as $\gamma \rightarrow 0$. In order to characterize this transition in the spectral statistics we must compute the localization length $L$ as a function of $\alpha$ and $b$.

\section{Estimates of the localization length}

In this section we estimate the localization length when $\alpha=0$. The argument also suggests the existence of a scaling relationship when $\alpha$ is non-zero, which is confirmed by numerical experiments described in section 4 .

To estimate the localization length $L$ at $\alpha=0$, we proceed as follows. We consider a vector $\boldsymbol{v}_{0}$ with one non-zero element, at index zero (we consider the indices of the infinite-dimensional vectors and matrices to run from $-\infty$ to $+\infty$ ):

$$
\left(v_{0}\right)_{i}=\delta_{i 0} \text {. }
$$

This vector can be written as a linear combination of eigenvectors $\phi_{j}$ of the matrix $\tilde{H}$ :

$$
\boldsymbol{v}_{0}=\sum_{j} a_{j} \boldsymbol{\phi}_{j}
$$

If the localization length is $L$, then we expect that approximately $L$ of the amplitudes $a_{j}$ are significant, and that most of the others are extremely small. Consider what happens when we multiply $v_{0}$ by $\tilde{H} N$ times:

$$
\boldsymbol{v}_{N}=\tilde{H}^{N} \boldsymbol{v}_{0}=\sum_{j} a_{j} E_{j}^{N} \boldsymbol{\phi}_{j} .
$$

When $N$ is large, the sum is dominated by values of $j$ for which $\left|E_{j}\right|$ is largest. For simplicity, let us assume that all but $L$ of the $a_{j}$ vanish: in this case, after a large number of multiplications the vector $v_{N}$ is approximately equal to a multiple of $\phi_{\max }$, the eigenvector corresponding to the largest eigenvalue $\left|E_{\max }\right|$. The number of multiplications required for $\phi_{\max }$ to become dominant is

$$
N^{*} \simeq E_{\max } /\left(\left|E_{\max }\right|-\left|E_{\text {next }}\right|\right) \simeq E_{\max } \rho
$$


where $E_{\text {next }}$ is the next largest of the $L$ contributing eigenvalues and $\rho$ is the density of states for these $L$ states. Since the distribution of eigenvalues is symmetric about $E=0$, the density of states is:

$$
\rho \simeq L /\left|E_{\max }\right| \text {. }
$$

The vector $v_{N}$ therefore becomes essentially a multiple of $\phi_{\max }$ when $N \gg N^{*} \simeq L$.

We can characterize the vector $v_{N}$ conveniently by considering the standard deviation $S(N)$ of the distribution of its elements $\left(v_{N}\right)_{i}$ :

$$
S(N)=\sum_{i} i^{2}\left(v_{N}\right)_{i}^{2} / \sum_{i}\left(v_{N}\right)_{i}^{2} \equiv \frac{B_{N}}{A_{N}} .
$$

The spread $S$ of the vector $v_{N}$ increases until $N \simeq N^{*}$, when $S(N)$ saturates at a value

$$
S\left(N^{*}\right) \simeq L \simeq N^{*} \text {. }
$$

The second approximate equality follows from (3.4) and (3.5). The strategy for calculating the localization length is to compute $S(N)$ using an alternative method which ignores the possibility of localization, and to solve (3.7) using this expression for $S(N)$. Taking account of the fact that some of the neglected coefficients $a_{j}$ will correspond to eigenvalues with magnitude greater than $E_{\max }$, we see that $S(N)$ continues to increase logarithmically after the break point $N^{*}$ instead of saturating, but this does not affect the equation (3.7) for the localization length.

The spread $S(N)$ can be estimated by considering the multiplication of $v_{0}$ by a string of $N$ different realizations of the random matrix ensemble, $\tilde{H}_{1} \ldots \tilde{H}_{N}$ :

$$
v_{N}^{\prime}=\tilde{H}_{N} \tilde{H}_{N-1} \ldots \tilde{H}_{2} \tilde{H}_{1} v_{0} \text {. }
$$

We can easily compute $S(N)$ for the vector $\boldsymbol{v}_{N}^{\prime}$ since all the random variables involved are independent. The spread of the vector $v_{N}$ would be expected to be similar to that of $v_{N}^{\prime}$ until the localization effects become apparent when $N \simeq N^{*}$, when the spread of $v_{N}^{\prime}$ continues to increase instead of saturating.

We now proceed to set up a recursion relation involving the second moment $S^{2}(N)$ of $v_{N}^{\prime}$, and to compute its value when $\alpha=0$. Because the matrix elements of the $\tilde{H}_{n}$ are random variables, we can regard the elements of the vectors $\boldsymbol{v}_{n}^{\prime}$ as random variables, with second moment $Y_{i}^{n}$ :

$$
Y_{i}^{n}=\left\langle\left(v_{n}^{\prime}\right)_{i}^{2}\right\rangle
$$

where \langle\rangle denotes an average over the random matrix ensemble. The matrix elements satisfy

$$
\left(\boldsymbol{v}_{n+1}^{\prime}\right)_{i}=\sum_{j}\left(\tilde{H}_{n}\right)_{i j}\left(\boldsymbol{v}_{n}^{\prime}\right)_{j}
$$

so that the $Y_{i}^{n}$ satisfy the recursion relation

$$
Y_{i}^{n+1}=\sum_{j=-b}^{b}\left(1+\delta_{i j}\right) Y_{j}^{n}+\alpha^{2} i^{2} Y_{i}^{n} .
$$

We consider the case in which $b$ is large. In this case we can represent the $Y_{i}^{n}$ by a function $Y_{n}(x)$, and approximate the sum in (3.11) by an integral:

$$
\begin{aligned}
Y_{n+1}(x) & =\int_{x-h}^{x+b} \mathrm{~d} x^{\prime} Y_{n}\left(x^{\prime}\right)+\alpha^{2} x^{2} Y_{n}(x) \\
& =\int_{-\infty}^{\infty} \mathrm{d} x \chi_{b}\left(x-x^{\prime}\right) Y_{n}\left(x^{\prime}\right)+\alpha^{2} x^{2} Y_{n}(x)
\end{aligned}
$$


where $\chi_{b}(x)$ is a characteristic function on an interval of width $2 b$. The sum, $A_{N}$ and second and fourth moments, $B_{N}, C_{N}$ of the variances are given by (c.f. (3.6)):

$A_{N}=\int_{-\infty}^{\infty} \mathrm{d} x Y_{N}(x) \quad B_{N}=\int_{-\infty}^{\infty} \mathrm{d} x x^{2} Y_{N}(x) \quad C_{N}=\int_{-\infty}^{\infty} \mathrm{d} x x^{4} Y_{N}(x)$.

We can use (3.13) to write down recurrence relations for the $A_{N}$ and $B_{N}$ : performing the $x$ integral in (3.12) and substituting (3.13) gives the following results.

$$
\begin{aligned}
& A_{N+1}=2 b A_{N}+\alpha^{2} B_{N} \\
& B_{N+1}=2 b B_{N}+\frac{2}{3} b^{3} A_{N}+\alpha^{2} C_{N} .
\end{aligned}
$$

When $\alpha=0$, taking the ratio of (3.15) to (3.14), and using the initial value $S^{2}(0)=0$, gives the result

$$
S^{2}(N)=\frac{B_{N}}{A_{N}}=\frac{1}{3} N b^{2}
$$

Using this result equation (3.7) gives $N^{*} \approx b^{2}$, so that

$$
L=\mathrm{O}\left(b^{2}\right)
$$

which is in agreement with numerical results obtained by Casati et al (1990).

We can also use this calculation to suggest a scaling relation for the localization length which applies when $\alpha \neq 0$ : we seek a scaling relation of the form

$$
L=b^{2} f(x)
$$

where $x$ is a scaling variable which is a function of $\alpha$ and $b$. The scaling function $f(x)$ is of order unity at $x=0$, and starts to change significantly around $x=1$. The following argumentent can be used to ideñtify the scaling variable $x$, but not the scáling function $f(x)$.

When $\alpha \neq 0$ (3.16) remains valid if the terms proportional to $\alpha^{2}$ in (3.14) and (3.15) can be neglected: approximating the ratio $S^{2}(N)=B_{N} / A_{N}$ using (3.16), we see that the condition for neglecting this term in (3.14) is

$$
N \ll N_{\mathrm{cr}}=\frac{1}{\alpha^{2} b} .
$$

Approximating the fourth moment $C_{N}$ using the square of the second moment, $C_{N} \simeq B_{N}^{2} / A_{N}$, we see that the same condition applies for neglecting the rightmost term in (3.15). The localization length is not significantly altered if $N_{\mathrm{cr}}$ (defined by (3.19)) is large compared to the break point $N^{*}$. The point at which $N_{\mathrm{cr}}=N^{*}$ corresponds to the point where the scaling variable is unity, i.e.

$$
x=1 \Leftrightarrow b^{2}=N^{*}=N_{\mathrm{cr}}=\frac{1}{\alpha^{2} b} .
$$

This shows that a suitable choice of scaling variable is $x=\alpha b^{3 / 2}$, so that our proposed scaling relation is of the form

$$
L=b^{2} f(x)=b^{2} f\left(\alpha b^{3 / 2}\right) .
$$

The argument above assumes that $N_{\mathrm{cr}} \gg 1$ and $N^{*} \gg 1$, as well as $b \gg 1$. When $\alpha$ is large, the first condition need not hold: using (3.19) we find that $N_{\mathrm{cr}}=1$ when $\alpha^{2} b \simeq 1$. This suggests that deviations from the scaling relation (3.21) will become apparent when $x \gg b$. In the limit of large $\alpha$ the problem can be analysed by treating the off-diagonal elements as a perturbation, but we will not pursue this further. 
It is interesting to note that, provided $\alpha$ is small enough for the scaling relation (3.21) for the localization length to hold, the parameter $\gamma$ describing the transition in the spectral statistics is a function of $x=\alpha b^{3 / 2}$ alone, $\gamma=x f(x)$, so that we have a one parameter scaling theory for the transition.

\section{Numerical experiments}

We have performed numerical experiments which verify these predictions, using matrices of dimension $\mathcal{N} \approx 5 b^{2}$, and eliminating states from the highest and lowest $15 \%$ of the spectrum to guard against finite-size effects. Figure 1 shows numerical measurements of the entropy localization length (Casati et al 1990), for various values of $b$, plotted to show the scaling function $f(x)$. In the region where $x \gg 1$, the function $f(x)=$ const. $x^{-2 / 3}$ (full curve in figure 1 ) is a good fit to the scaling function, at least up to $x=20$. The results follow the scaling curve up to a break point, which increases as $b \rightarrow \infty$ : deviations from the scaling curve are already visible for the $b=3$ data in figure 1 .

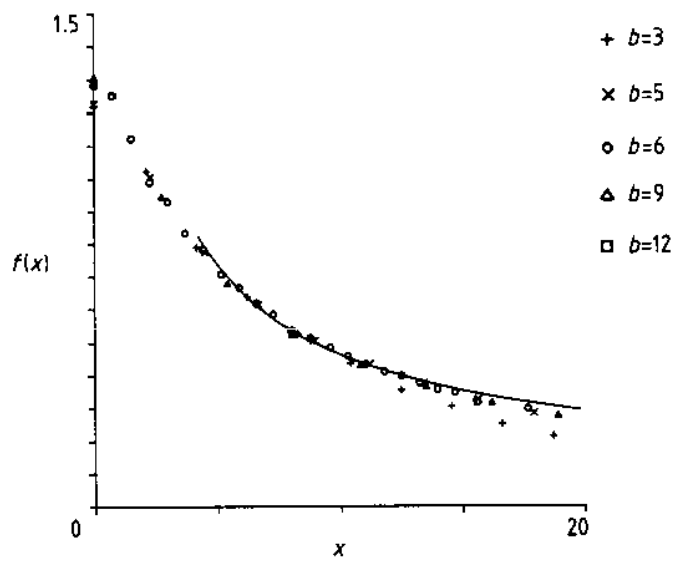

Figure 1. Plot showing one parameter scaling function $f(x)$ for localization length, defined by $L=b^{2} f(x), x=\alpha b^{3 / 2}$ : the different styles of point indicate different values of $b$. The points deviate from the scaling relation for large $x$, but the break point where the deviations begin increases as $b \rightarrow \infty$.

We also investigated the transition from Poisson to Wigner statistics as $\gamma$ increases by calculating a histogram of the level spacing distribution $P(S)$, and performing a least-squares fit to a Brody distribution (Brody 1973), which interpolates between the Poisson and Wigner distributions as a parameter $q$ is increased from 0 to 1 . For these numerical experiments we used a sample of at least 125 matrices of dimension 800 for each point, and the bin size used for computing the histogram was 0.1 . The results are shown in figure 2: most of the data lie on a scaling curve, implying that the level spacing distribution is a function of the parameter $\gamma(x)$ only. The deviations from the scaling curve are due to finite-size effects: for large values of $b$ the localization length can be comparable to the dimension of the matrix and it is not possible to get a Poissonian level spacing distribution, which requires the superposition of many independent spectra. Usually the fit to the Brody distribution was very good as judged by eye (see figure 3 ), but we do not claim that the fit is statistically significant. 


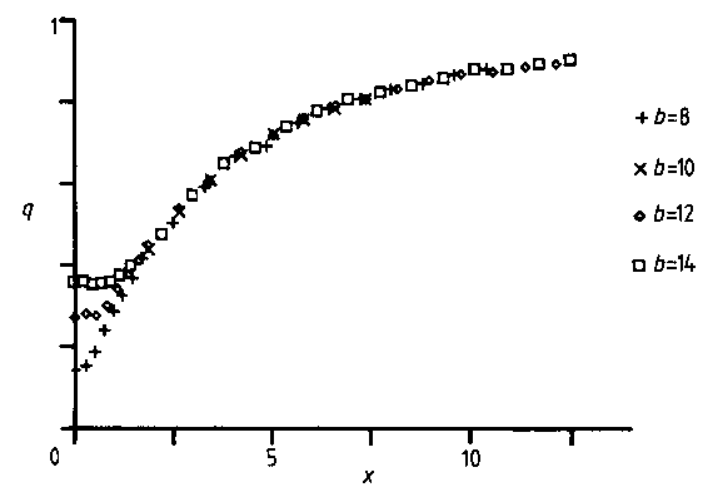

Figure 2. The parameter $q$ characterizing a fit of the Brody distribution to the level spacing distribution, plotted as a function of the scaling parameter $x$. Different styles of point indicate different values of $b$. The deviations at small $q$ and large $b$ are a finite-size effect, and are discussed in the text.

\section{Discussion}

We have argued that the structure of the matrices of our ensemble mimics that of the matrix encountered when computing one chaotic quantum Hamiltonian in the eigenbasis of another such Hamiltonian. Also, we have shown that the model exhibits a transition in the form of its spectral statistics, rather than displaying the universal GOE statistics observed in chaotic quantum systems. We should therefore consider the reasons why the transitional spectral statistics are not observed in chaotic systems.

One possibility is that $\gamma$ is always large, so that GOE statistics are expected after all. We can use semiclassical estimates of the density of states $\rho$ and typical size of matrix elements $\sigma$, to compute how the transition parameter $\gamma$ scales as $\hbar \rightarrow 0$ (it is only in this limit that the spectrum becomes dense, so that we can compute well-defined statistics). These estimates give $\rho=\mathrm{O}\left(\hbar^{-d}\right)$ and $\sigma=\mathrm{O}\left(\hbar^{(d-1) / 2}\right)$ (Wilkinson 1987,

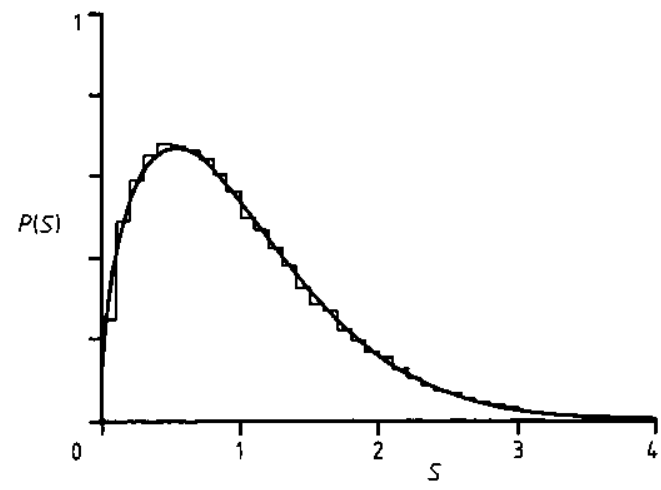

Figure 3. A typical level spacing distribution, showing the fit to a Brody distribution: the data are for $b=10, x=2.26$, and the fitted value of $q$ is 0.484 . 
Feingold, Leitner and Piro 1989), so that $\alpha=\mathrm{O}\left(\hbar^{(d+1) / 2}\right), b=\mathrm{O}\left(\hbar^{1-d}\right), x=\alpha b^{3 / 2}=$ $\mathrm{O}\left(\hbar^{2-d}\right)$, where $d$ is the number of degrees of freedom. It follows that $\gamma(x) \rightarrow \infty$ as $\hbar \rightarrow 0$, implying GOE statistics, only if $d>2$. In the important case when $d=2$ semiclassical considerations do not constrain $\gamma$ to be large. We must conclude that the observed GOE statistics are explained by differences between our model and the matrix elements of a semiclassical system.

The method we use for estimating the localization length is clearly insensitive to many features of the model, provided the matrix elements are independent: for instance it does not matter if the matrix is not strictly banded (the matrix elements only have to decay rapidly away from the diagonal), and it does not matter whether or not their distribution is Gaussian. We conclude that the matrix elements of semiclassical systems must be subtly correlated. This is consistent with earlier work in which correlations between matrix elements were used to explain deviations of semiclassical spectra from the GOE model (Wilkinson 1988).

In conclusion, we have investigated the scaling properties of the localization length in a banded random matrix ensemble, and we have shown that localization gives rise to a transition in the spectral statistics of this model, depending on a single scaling parameter. We have commented on the similarity of the matrices to those occurring in the quantum mechanics of chaotic systems, and shown that the results of using a random matrix to model matrix elements of these systems may be misleading.

\section{Acknowledgments}

We wish to thank Dr W Swiatecki for many useful discussions. MF thanks J M Deutsch for suggesting the model used in this paper. DML acknowledges the support of $J \mathrm{D}$ Doll. This work was supported in part by the Director, Office of Energy Research, Office of High Energy and Nuclear Physics, Division of Nuclear Physics, and Office of Basic Energy Sciences Research, Engineering and Geoscience Division of the U.S. Department of Energy under Contract No. DE-AC03-76SF00098, and by the U.S. National Science Foundation under Grant No. NSF-PYI-84-51276.

\section{References}

Berry M V 1977 J. Phys. A: Math. Gen. 10 2083-91

Bohigas O, Giannoni M J and Schmidt C 1984 Phys. Rev. Lett. 52 1-4

Brody T A 1973 Nuovo Cimento Lett. 7482

Casati G, Molinari L and Izraelev F M Phys. Rev. Lett. $641 \overline{8} 52-5$

Chirikov B V, Izraelev F M and Shepelyanski D L 1981 Sov. Sci. Rev. C2 209

Feingold M, Leitner D M and Piro O 1989 Phys. Rev. A 396507

Feingold M, Leitner D M and Wilkinson M 1990 Phys. Rev. Lett. submitted

Feingold $M$ and Peres A 1986 Phys. Rev. A 34591

Heller E J 1984 Phys. Rev. Lett. 53 1515-9

Ishii K 1973 Prog. Theor. Phys. Suppl. (Japan) 5377

Johnson $\mathrm{R}$ and Kunz $\mathrm{H} 1983$ J. Phys. C: Condens Matter 163895

Molcanov S A 1981 Commun. Math. Phys. 78 429-46

Porter C E (ed) 1965 Statistical Theories of Spectra: Fluctuations (New York: Academic)

Sebr P 1990 Phys. Rev. Lett. 64 1855-8

Wigner E P 1955 Ann. Marh. 62145

Wilkinson M 1987 J. Phys. A: Marh. Gen. 20 2415-23

1988 J. Phys. A: Math. Gen. 21 1173-90 